\title{
Molecular Markers for Species Identification of Hessian Fly Males Caught on Sticky Pheromone Traps
}

\author{
MING-SHUN CHEN,${ }^{1,2,3}$ SHANDA WHEELER,${ }^{2}$ HOLLY DAVIS, ${ }^{2}$ R. JEFF WHITWORTH, ${ }^{2}$ \\ ALLEN KNUTSON,${ }^{4}$ KRISTOPHER L. GILES,${ }^{5}$ TOM A. ROYER,${ }^{5}$ AND MARGARET SKINNER ${ }^{6}$
}

\begin{abstract}
J. Econ. Entomol. 107(3): 1110-1117 (2014); DOI: http://dx.doi.org/10.1603/EC13384
ABSTRACT Pheromone traps have been widely used to monitor insect population activity. However, sticky pheromone traps for the Hessian fly (Mayetiola destructor), one of the most destructive pests of wheat, have been used only in recent years. Hessian fly male adults are small and fragile, and preserving specimens during sorting of sticky pheromone traps is a challenge when intact specimens are often required to visually distinguish them from related insects such as fungus gnats. In this study, we have established a quick and reliable method based on polymerase chain reaction markers to correctly distinguish Hessian fly males from other closely related insects. Two Hessian fly-specific markers were established, one based on the trypsin gene MDP-10 and the other based on a gene encoding the salivary gland protein SSGP31-5. Both markers provided $>98 \%$ identification success of 110 Hessian fly samples prepared from single insects. The method should provide a useful tool to allow for identification of Hessian fly individuals on sticky pheromone traps or in other situations when Hessian fly eggs, larvae, pupae, and adults are difficult to distinguish from other insects.
\end{abstract}

KEY WORDS Mayetiola destructor, hessian fly, pheromone trap, wheat, molecular marker

The Hessian fly, Mayetiola destructor, is a major pest of wheat in the United States and elsewhere worldwide (Buntin 1999, Pauly 2002, Stuart et al. 2012). After hatching from eggs deposited on leaves, neonates migrate along the leaf and enter the plant between the leaf sheath and stem, where they establish a single feeding site on the stem. Hessian fly larvae manipulate host plants extensively, presumably by injecting salivary secretions into wheat tissue (Byers and Gallun 1971, Chen et al. 2010). A single larva can irreversibly inhibit wheat growth, suppress wheat defenses, and establish a permanent feeding site (Byers and Gallun 1971, Liu et al. 2007). Unless a new tiller(s) grows out, the infested plant dies after Hessian fly larvae pupate and stop feeding.

Because Hessian fly larvae live within wheat plant tillers and damage is irreversible, the most effective control measures are preemptive (late planting dates, insecticidal seed treatments, and/or resistant cultivars). The Hessian fly interacts with host plants, much

\footnotetext{
${ }^{1}$ Hard Winter Wheat Genetics Research Unit, Center for Grain and Animal Health Research, USDA-ARS, 1515 College Ave., Manhattan, KS 66502.

${ }^{2}$ Department of Entomology, Kansas State University, 123 Waters Hall, Manhattan, KS 6506.

${ }^{3}$ Corresponding author, e-mail: mchen@ksu.edu or ming-shun.chen@ ars.usda.gov.

4 Texas A\&M AgriLife Extension Service, 17360 Coit Rd., Dallas, TX 75252 .

${ }^{5}$ Department of Plant Pathology and Entomology, Oklahoma State University, 127 NBC, Stillwater, OK 74078.

${ }^{6}$ Entomology Research Laboratory, University of Vermont, 661 Spear St., Burlington, VT 05405-0105.
}

like pathogens, in a typical gene-for-gene relationship, and plant resistance to Hessian fly larvae is categorized as antibiosis because first instars die in resistant plants without development (Stuart et al. 2012). Plant protection based on deployment of a specific resistant gene, however, is estimated to last $6-8$ yr because Hessian fly populations change rapidly over time (Gould 1998, Chen et al. 2009). Long-term successful deployment of resistant genes requires continuous monitoring of Hessian fly populations in different geographic regions and identification of the genes that remain effective. Currently only around $25-40 \%$ of wheat cultivars have Hessian fly resistance, depending on the wheat-growing region.

Several additional control measures are commonly used to manage Hessian fly, including late planting (called the Best Pest Management Planting Date, BPMPD) and application of insecticides (Buntin and Bruckner 1990; Buntin et al. 1990, 1992; Buntin 1992; Morgan et al. 2005). Both of these tactics require accurate monitoring or prediction of Hessian fly population dynamics to be effective. The BPMPD approach recommends planting wheat after adult Hessian flies' activity has ceased (Whitworth et al. 2010). However, owing to annual fluctuations in temperature and rainfall, the BPMPD must be determined annually by monitoring Hessian fly populations. The proper timing for application of insecticides is based on adult activity that needs to be documented as well. Sampling of Hessian fly pupae (flaxseeds), larvae, or eggs in the field is very time-consuming, whereas monitoring adult activity using sticky pheromone traps is poten- 
tially a more efficient method for individual fields and among fields in an area-wide program.

Like many other insects, Hessian fly females emit a sex pheromone to attract males (Andersson et al. 2009). A synthetic pheromone for Hessian fly has been produced and is commercially available (Anderson et al. 2012). In recent years, pheromone lures placed on sticky cards have been used to monitor Hessian fly populations in the United States. Initial results from trappings have revealed unexpected phenomena: 1) large numbers of flies are caught in late fall, even after the previously reported fly-free dates; 2) the population level of flies based on sticky pheromone trap results is not well correlated with observed field damage; and 3) large numbers of flies captured on sticky pheromone traps do not necessarily predict subsequent significant infestations in the same areas (R.J.W. et al., unpublished data). These conflicting observations and concerns about variable levels of taxonomic expertise among scouts identifying flies in traps highlight potential issues with the usefulness of Hessian fly sticky pheromone traps. Indeed, these observations raise the question whether the insects caught on sticky pheromone traps are in fact Hessian fly or are related species that look similar, such as other Mayetiola species and fungus gnats (Gagne 1975). Male Hessian flies are difficult to distinguish morphologically from related species, especially after the insects get entangled in the trap glue. The objectives of this research are 1) establish a molecular method that can distinguish quickly and reliably Hessian fly males from morphologically similar species captured in sticky pheromone traps, and 2) examine if insects captured in Hessian fly sticky pheromone traps and visually identified as Hessian flies are indeed Hessian fly males.

\section{Materials and Methods}

Pheromone Traps. Capsules or lures of Hessian fly pheromone were obtained from PheroNet (Alnarp, Sweden). The Hessian fly sex pheromone lure was loaded onto a 10-mm polyethylene dispenser and placed in the center of a sticky trap (Trécé Inc., Adair, OK). At multiple wheat fields in Kansas, Texas, Oklahoma, and Vermont, one to three of these sticky traps were placed on the field border and baited with a pheromone lure over several sampling dates in the fall and/or spring (2010-2012, see Table 2). The traps were hung on bamboo sticks $\approx 30 \mathrm{~cm}$ from the surface of the soil. The numbers of captured Hessian fly adults were counted weekly, and the sticky liner and lure were replaced. The sticky liners were placed individually in 1-gallon plastic bags and stored in a freezer $\left(-20 \pm 2^{\circ} \mathrm{C}\right)$ until they could be processed.

Prescreening of Hessian Fly Male Adults Based on Morphology. Hessian fly male adults were visually separated from other dipterans under a scope (6$20 \times$ ) based on morphological and taxonomic characteristics as outlined in Diagnostic Methods for Hessian Fly Mayetiola destructor (PaDIL-Plant Biosecurity Toolbox 2010). In addition, emerged males from a Kansas population maintained in a greenhouse were collected and used as reference specimens to aid in identification.

Fungus Gnats. Adult darkwinged fungus gnats ( $\mathrm{Bra}$ dysia sp., Family Scaridae) were collected from an indoor plant growing room at the Entomology Research Laboratory, University of Vermont, Burlington, VT, where green beans are grown continuously for a thrips laboratory colony. The humid conditions favor a small continuous population of fungus gnats, which feed on organic matter in the potting soil. Live adult flies were collected in a net and placed immediately in $95 \%$ ethyl alcohol.

Mayetiola hordei. M. hordei (also called the barley midge) is similar to Hessian fly morphologically (Bouktila et al. 2006) and genetically according to their genome sequences. Therefore, a DNA sample from a pool of $M$. hordei adult flies was used as a negative control. $M$. hordei flies were collected from a greenhouse culture by Dr. Mustapha El Bouhssini at the International Center for Agricultural Research in the Dry Areas, Aleppo, Syria; placed in 95\% ethyl alcohol; and shipped to the United States for DNA extraction.

DNA Extraction. Hessian flies were either collected from laboratory cultures or obtained from traps using forceps. Insects were put individually into 1.5-ml Eppendorf tubes with $100 \mu \mathrm{l}$ STE buffer (10 mM Tris$\mathrm{HCl}, 1 \mathrm{mM}$ EDTA, pH 8.0, 0.1M NaCl) and homogenized with an electric microtube pestle. Each sample was then incubated in boiling water for $5 \mathrm{~min}$ and centrifuged at 12,000 rpm for $5 \mathrm{~min}$. The supernatant from each sample was collected and placed in a new Eppendorf tube, to which $250 \mu \mathrm{l}$ of $-20^{\circ} \mathrm{C}$ ethanol was added, and the tube was inverted several times to mix the contents. The samples were incubated at $-20^{\circ} \mathrm{C}$ for $14-18 \mathrm{~h}$ and then centrifuged at full speed for 20 min at $4^{\circ} \mathrm{C}$ to collect a DNA pellet, after which the supernatant was discarded. The pellets were reconstituted with $30 \mu \mathrm{l}$ of $\mathrm{ddH}_{2} 0$, vortexed thoroughly, and stored at $-20^{\circ} \mathrm{C}$ for short-term storage or $-80^{\circ} \mathrm{C}$ for long-term use.

DNA samples from insect populations (Kansas laboratory Hessian flies and Vermont fungus gnats) were extracted in the same way, but each sample was from a pool of 10 individuals instead of single insects.

Primer Design and Polymerase Chain Reaction Amplification. Primers for polymerase chain reaction (PCR) amplification were designed using the Beacon Designer software (version 7) with default parameters and synthesized by Integrated DNA Technologies (Coralville, IA). Full target gene sequences were used to select all possible primer combinations. From those combinations, primer pairs with significant sequence variations among Hessian fly, M. hordei, and fruit fly sequences were selected for synthesis. PCR amplification was carried out according to the following program: $60 \mathrm{~s}$ at $94^{\circ} \mathrm{C}$ for DNA denaturation, $60 \mathrm{~s}$ at $55^{\circ} \mathrm{C}$ for annealing, and $120 \mathrm{~s}$ at $72^{\circ} \mathrm{C}$ for polymerase extension. For the common marker (CM, actin, AF017427) and Hessian-fly specific marker 2 (HFSM2, SSPG31-5, EV466578), 35 cycles were carried out for PCR amplification, whereas for Hessian-fly specific 


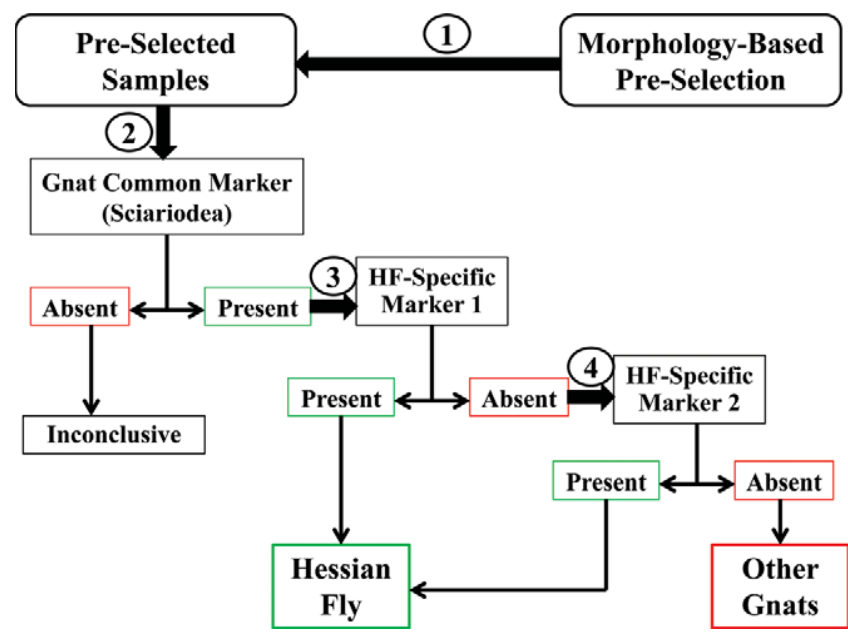

Fig. 1. Overall strategy to identify Hessian fly males on a sticky pheromone trap. The numbers within the circles above or beside a major arrow indicate four major steps in the identification process: 1) morphology-based preselection to exclude apparent nonmidges, 2) Mycetophiloidea-common marker selection to exclude nonmidge insects with similar morphology, 3) selection by Hessian fly-specific marker 1 to exclude non-Hessian fly midges, and 4) final selection by Hessian fly-specific marker 2 to reduce misidentification by specific marker 1 due to errors or gene sequence variation.

marker 1 (HFSM1, MDP10, AEGA01028834), 45 cycles were carried out. For marker specificity testing, different annealing temperatures of $35,40,45,50$, and $55^{\circ} \mathrm{C}$ were tested, with other conditions remaining the same. A negative control without DNA template was carried for each batch of PCR. Different annealing temperatures were tested for each primer pair. A negative result for a specific PCR was repeated an additional time. If both PCR repeats were negative, the sample was considered negative for this primer pair. The DNA fragments were separated on $3 \%$ agarose gels containing $0.5 \mu \mathrm{g} / \mathrm{ml}$ ethidium bromide, ran at $80 \mathrm{~V}$ for $60 \mathrm{~min}$, and DNA fragment bands were visualized using a Bio Doc-It System (UVP, Upland, CA).

DNA Sequencing. Representative DNA fragments from PCR were electrophoresized on 3\% agarose gels in TAE buffer. DNA bands with expected sizes were extracted from the gel with a GeneClean Kit II (Bio 101, LA Jolla, CA). The purified DNA fragments were sequenced directly using one of the PCR primers in the Gene Sequencing Center at Kansas State University, Manhattan, KS.

\section{Results and Discussion}

The overall strategy for accurate identification of Hessian flies on sticky pheromone traps is outlined in Fig. 1. Once a sticky pheromone trap is brought back from the field, putative Hessian fly males are preselected based on morphology, as described in the Materials and Methods section. Approximately $10-40 \%$ of insects on a trap, depending on time and location of the trap, can be easily identified as non-Hessian fly owing to distinct morphology or size. The second step is to use a molecular marker that is common to the Hessian fly and related species from the same superfamily Mycetophiloidea. This common marker serves as a positive control to eliminate negative results due to bad sample preparation or mistakes from the first step (visual examination) due to wrong identification because of damaged insects. A negative result will eliminate the sample from further analysis. The third step is to use a Hessian fly-specific marker to screen positive samples from Step 2. If the Hessian fly-specific marker gave a positive result, then the insect was counted as a Hessian fly male. If this Hessian flyspecific maker was negative, the sample was further analyzed with a second Hessian fly-specific marker to avoid errors due to Hessian fly population heterogeneity. If the second Hessian fly-specific marker was positive, the insect was counted as a Hessian fly male. If the marker was negative, the insect was counted as a non-Hessian fly male.

Marker Design. The gene encoding actin isoform A was chosen as the CM because a pairwise comparison between orthologs revealed that this gene is the most conserved among the genes we analyzed, including ribosomal S13 and glyceraldehyde-3-phosphate dehydrogenase among the members of the Mycetophiloidea superfamily (data not shown). The nucleotide sequences of the Hessian fly actin gene within the primer regions are identical to those of the corresponding M. hordei (Fig. 2A, Mho) actin gene, and also share $86.3 \%$ identity with the actin genes of Aedes albopictus (Aal, DQ657949) and Drosophila melanogaster (Dme, BT099815. The PCR target region is located in the middle toward the $5^{\prime}$-coding region and the expected PCR product size is $148 \mathrm{bp}$.

Hessian fly-specific marker 1 (HFSM1) was developed based on the presence of the trypsin gene MDP10 in the Hessian fly genome, but sequences with detectable similarity at the nucleotide level could not be found in the genomes of other insects through blasting with default setting (Chen et al. 2013). Specifically, 
A

\author{
PCR Location \\ Product Size
}

Sequence
Similarity

Primer pair

\section{Common Marker (The Actin A Gene)}

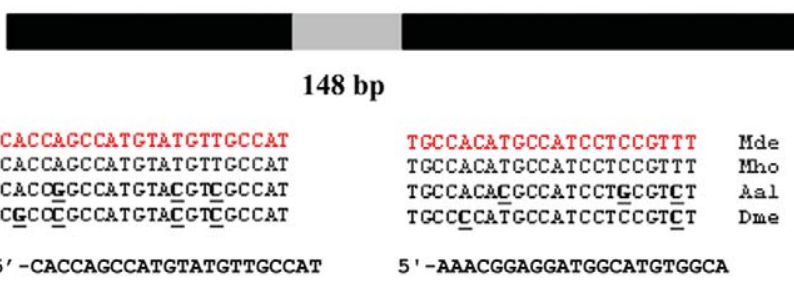

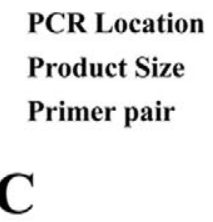

\section{B}

\section{PCR Location \\ Product Size \\ Primer pair}

\section{Hessian Fly-Specific Marker 1 (The MDP10 Gene)}

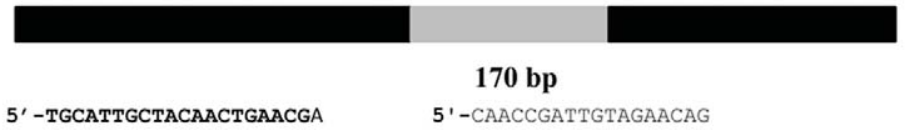

\section{Hessian Fly-Specific Marker 2 (The SSGP31-5 Gene)}

\section{$369 \mathrm{bp}$}

Fig. 2. Primer design and sequences for the midge/gnat common marker, Hessian fly-specific marker 1, and Hessian fly-specific marker 2. (A) Primers for the midge/gnat common marker based on the gene encoding actin isoform. The gray portion in the black bar is the location of the PCR product. Mde, Mho, Aal, and Dme represent Mayetiola destructor, M. hordei, Aedes albopictus, and Drosophila melanogaster, respectively. The alignment shows the sequences in the two primer regions are identical between Mde and Mho, but have several different residues in the Aal and Dme genes. The actual primer sequences are shown under the alignment. (B) Primers for the Hessian fly-specific marker 1 based on the trypsin MDP10 gene (Chen et al. 2013). (C) Primers for the Hessian fly-specific marker 2 based on the SSGP31-5 gene (Chen et al. 2010).

BLASTN search with the nucleotide sequence of the Hessian fly MDP10 gene with default settings revealed no hit with sequences in GenBank. A similar BLASTN search against a local database that contains a draft $M$. hordei genome sequence revealed no hit as well (data not shown). Accordingly, a pair of primers targeting a segment located in the middle of the coding region toward the $3^{\prime}$-end of the MDP10 gene were selected and used for PCR (Fig. 2B). The expected PCR product is $170 \mathrm{bp}$.

Hessian fly-specific marker 2 (HFSM2) was designed according to the SSGP31-5 gene, which encodes a secretory salivary protein (Chen et al. 2010). Again the nucleotide sequence of the Hessian fly SSGP31-5 gene shares no identity based on BLASTN comparison with any gene in the draft $M$. hordei ge-
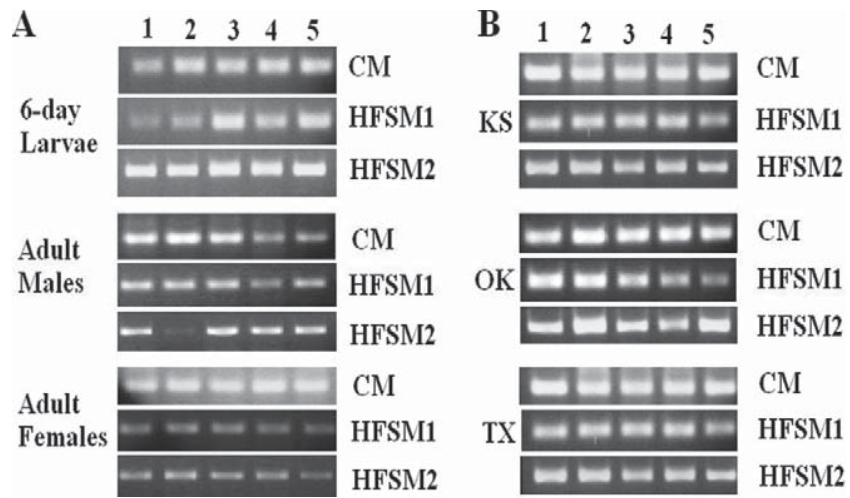

Fig. 3. PCR amplification of DNA samples extracted from individual Hessian flies with different primer sets. (A) Marker testing with five insects each from different developmental stages. CM, HFSM1, and HFSM2 represent primers for the midge/gnat common marker, Hessian fly-specific marker 1, and Hessian fly-specific marker 2, respectively. (B) Marker testing with DNA samples from five individual Hessian fly males from different regional populations. KS, OK, and TX represent a fly population from Kansas, Oklahoma, and Texas, respectively. 


\section{$\begin{array}{lllll}55 & 50 & 45 & 40 & 35^{\circ} \mathrm{C}\end{array}$ \\ CM

\begin{tabular}{|l}
\hline$+5 e s s i a n$ fly \\
Barley midge \\
Fungus gnat
\end{tabular}
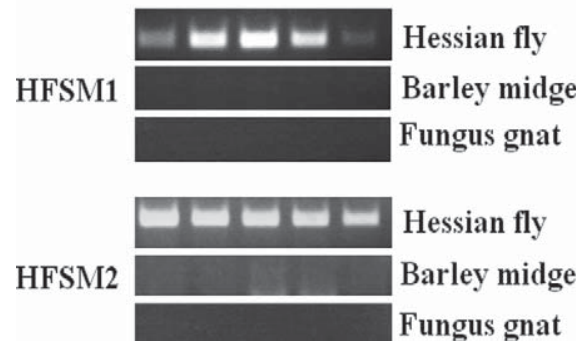

Fig. 4. PCR amplification at five temperatures of DNA samples obtained from three different species, the Hessian fly, M. hordei, and fungus gnat, with primer pairs for the common marker (CM), Hessian fly-specific marker 1 (HFSM1), and Hessian fly-specific primer 2 (HFSM2).

nome sequence or with any known sequences in GenBank (data not shown). A pair of primers were designed to cover the whole coding region of the SSGP31-5 gene (Fig. 2C). The expected size of PCR product is $369 \mathrm{bp}$.

Marker Testing With Known Hessian Fly Individuals. To determine the usefulness of the designed PCR markers for the identification of Hessian flies, we first tested the markers with known Hessian fly individuals from a Kansas population maintained in the greenhouse (Chen et al. 2009). DNA samples were extracted from individual 6-d larvae, adult males, and adult females, separately. The DNA samples were then subjected to PCR analysis with primer pairs for common (CM) and Hessian fly-specific (HFSM1 and HFSM2) markers (Fig. 3A). All markers detected expected PCR products in all individual insects, indicating that the markers are useful for Hessian fly detection. We further tested the effectiveness of the markers for recognizing Hessian fly males on sticky pheromone traps. DNA samples were extracted from five males from each pheromone trap collected from Kansas, Oklahoma, or Texas. Primer pairs for common and Hessian fly-specific markers were able to detect specific PCR fragments from insects on sticky traps (Fig. 3B).
Marker Specificity Testing. To determine if the combinations of common and Hessian fly-specific markers can distinguish the Hessian fly from its related species, DNA samples were prepared from Kansas Hessian fly males, M. hordei, and fungus gnats, and subjected to PCR analysis. As shown in Fig. 4, the primer pair for the CM marker detected a strong band with the expected size at annealing temperatures ranging from 35 to $55^{\circ} \mathrm{C}$, even though the band intensity decreased slightly at $40^{\circ} \mathrm{C}$ and significantly at $30^{\circ} \mathrm{C}$. In contrast, the primer pairs for the two Hessian flyspecific markers, HFSM1 and HFSM2, could only produce expected PCR fragments from the Hessian fly sample. No apparent DNA bands with expected sizes were obtained from the $M$. hordei or fungus gnat samples. There was a weak band in the $M$. hordei sample at annealing temperatures of 40 and $45^{\circ} \mathrm{C}$ with the primer pair for HFSM2, but the size of the band was much smaller than expected, and the band disappeared at other temperatures.

After the initial success, we further tested individual Hessian fly males from three different populations: a Kansas Hessian fly population collected from Scott County in 2005 and maintained in the greenhouse thereafter (Chen et al. 2009), Hessian fly biotype L (Sosa 1978), and an Israeli Hessian fly population (Johnson et al. 2012). The PCR success rates were 97-100\% for CM, $98-100 \%$ for HFSM1, and $80-100 \%$ for HFSM2 (Table 1). The PCR detection rates with 20 DNA samples from fungus gnats were $100 \%$ for CM, but $0 \%$ for the two Hessian fly-specific markers. These observations indicated that the PCR marker combinations can effectively distinguish Hessian fly males from related gnats.

Identification of Hessian Flies on Sticky Pheromone Traps. After the conditions for specific identification of Hessian fly individuals were established for the common and specific markers, the standard procedure was applied for the analysis of large numbers of insects obtained from Hessian fly sticky pheromone traps. In all, 353 insects preidentified based on morphology as Hessian fly males from sticky pheromone traps collected at different locations in Kansas, Oklahoma, Texas, and Vermont were analyzed using the common marker CM (Table 2, left part). DNA samples from 318 of these insects were successfully amplified by the PCR marker primers for CM, yielding a success rate of $90 \%$. The success rates with primers for $\mathrm{CM}$

Table 1. Number of preidentified Hessian fly males, based on morphology, from three geographic populations and fungus gnat (Bradysia sp.) males identified as positive for Hessian fly using a common and two specific markers

\begin{tabular}{|c|c|c|c|c|c|c|c|}
\hline \multirow{2}{*}{ Insect } & \multirow{2}{*}{ Sample size } & \multicolumn{2}{|c|}{$\mathrm{CM}$} & \multicolumn{2}{|c|}{ HFSM1 } & \multicolumn{2}{|c|}{ HFSM2 } \\
\hline & & Positive & $\%$ & Positive & $\%$ & Positive & $\%$ \\
\hline Kansas Hessian fly & 100 & 97 & 97 & 98 & 98 & 98 & 98 \\
\hline Biotype L & 5 & 5 & 100 & 5 & 100 & 5 & 100 \\
\hline Israeli Hessian fly & 5 & 5 & 100 & 5 & 100 & 4 & 80 \\
\hline Fungus gnat & 20 & 20 & 100 & 0 & 0 & 0 & 0 \\
\hline
\end{tabular}

CM, HFSM1, and HFSM2 represent the common marker for midges and gnats based on the actin gene, the Hessian fly-specific marker 1 based on the trypsin MDP10 gene, and the Hessian fly-specific marker 2 based on the SSGP31-5 gene, respectively.

Fungus gnats were used as negative controls for the Hessian fly-specific markers. 
Table 2. Number of morphologically preidentified Hessian fly males in sticky pheromone traps verified as Hessian fly using the midge/gnat common marker and two Hessian fly-specific markers

\begin{tabular}{|c|c|c|c|c|c|c|c|c|}
\hline \multirow{2}{*}{ Trap location } & \multirow{2}{*}{ Trap date } & \multirow{2}{*}{ No. of insects } & \multicolumn{2}{|c|}{$\underline{\text { Common markers }}$} & \multicolumn{4}{|c|}{ Hessian fly-specific markers } \\
\hline & & & Positive & $\%$ & HFSM1 & HFSM2 & Total & $\%$ \\
\hline Total of all samples & & 353 & 318 & 90 & 306 & 3 & 309 & 97 \\
\hline Kansas & & 90 & 88 & 98 & 87 & 0 & 87 & 99 \\
\hline DK & 14 Oct. 2010 & 5 & 5 & 100 & 5 & - & 5 & 100 \\
\hline DK & 20 April 2011 & 25 & 25 & 100 & 25 & - & 25 & 100 \\
\hline DK & 7 Oct. 2010 & 5 & 3 & 60 & 3 & - & 3 & 100 \\
\hline DK-1 & 26 April 2012 & 5 & 5 & 100 & 5 & - & 5 & 100 \\
\hline DK-2 & 27 May 2012 & 5 & 5 & 100 & 5 & - & 5 & 100 \\
\hline DK-2 & 26 April 2012 & 25 & 25 & 100 & 24 & 0 & 24 & 96 \\
\hline DK-1 & 28 April 2011 & 20 & 20 & 100 & 20 & - & 20 & 100 \\
\hline Oklahoma & & 15 & 9 & 60 & 9 & - & 9 & 100 \\
\hline Marland & 13 April 2012 & 5 & 5 & 100 & 5 & - & 5 & 100 \\
\hline Perkins & 17 April 2012 & 5 & 1 & 20 & 1 & - & 1 & 100 \\
\hline Perkins 2 & 17 April 2012 & 5 & 3 & 60 & 3 & - & 3 & 100 \\
\hline Texas & & 188 & 175 & 93 & 172 & 3 & 175 & 100 \\
\hline Hill Co bb & 11 Nov. 2011 & 5 & 5 & 100 & 5 & - & 5 & 100 \\
\hline Hill Co bb-2 & 11 Nov. 2011 & 5 & 4 & 80 & 3 & 1 & 4 & 100 \\
\hline Cooke Co F4 & 26 Oct. 2011 & 5 & 5 & 100 & 5 & - & 5 & 100 \\
\hline Cooke Co F4-2 & 26 Oct. 2011 & 5 & 5 & 100 & 5 & - & 5 & 100 \\
\hline Dorchester RR B3 & 8 Nov. 2011 & 5 & 5 & 100 & 5 & - & 5 & 100 \\
\hline Cook Co & Year 2012 & 5 & 5 & 100 & 5 & - & 5 & 100 \\
\hline Cook Co F3 & 25 Oct. 2011 & 5 & 5 & 100 & 5 & - & 5 & 100 \\
\hline Grayson Co F2 & 8 Nov. 2011 & 3 & 3 & 100 & 3 & - & 3 & 100 \\
\hline Grayson Co & 15 Nov. 2011 & 5 & 3 & 60 & 3 & - & 3 & 100 \\
\hline Grayson Co F2 & 22 Nov. 2011 & 5 & 2 & 40 & 2 & - & 2 & 100 \\
\hline $\mathrm{F} 6$ & Year 2012 & 5 & 5 & 100 & 5 & - & 5 & 100 \\
\hline Hill Co varietal A & 30 Mar. 2012 & 5 & 5 & 100 & 5 & - & 5 & 100 \\
\hline Hill Co gas well & 8 Mar. 2012 & 5 & 5 & 100 & 5 & - & 5 & 100 \\
\hline Lindsey field4 & 8 Nov. 2011 & 5 & 5 & 100 & 5 & - & 5 & 100 \\
\hline Hillsboro gas st & 3 Nov. 2011 & 5 & 5 & 100 & 5 & - & 5 & 100 \\
\hline Dorchester field2 & 8 Nov. 2011 & 5 & 5 & 100 & 5 & - & 5 & 100 \\
\hline Hill Co triangle & 14 Mar. 2012 & 5 & 4 & 80 & 4 & - & 4 & 100 \\
\hline Dorchester RR B4 & 8 Nov. 2011 & 5 & 4 & 80 & 4 & - & 4 & 100 \\
\hline Hill Co gas well & 15 Mar. 2012 & 5 & 5 & 100 & 5 & - & 5 & 100 \\
\hline Dorchester RR & 28 Feb. 2012 & 5 & 5 & 100 & 5 & - & 5 & 100 \\
\hline Cooke Co F2 & 10 April 2012 & 5 & 5 & 100 & 5 & - & 5 & 100 \\
\hline Cooke Co & 10 April 2012 & 5 & 5 & 100 & 5 & - & 5 & 100 \\
\hline RR & 10 April 2012 & 5 & 5 & 100 & 5 & - & 5 & 100 \\
\hline Hill Co old airport & 30 Mar. 2012 & 5 & 5 & 100 & 4 & 1 & 5 & 100 \\
\hline Hill Co old airport & 15 Mar. 2012 & 5 & 4 & 80 & 4 & - & 4 & 100 \\
\hline Hill Co 4CR 2340 & 14 Mar. 2012 & 5 & 5 & 100 & 5 & - & 5 & 100 \\
\hline Grayson Co F2 & 4 April 2012 & 5 & 5 & 100 & 5 & - & 5 & 100 \\
\hline Hill Co bb & 13 April 2012 & 5 & 5 & 100 & 5 & - & 5 & 100 \\
\hline Hill Co old airport & 17 Nov. 2011 & 5 & 4 & 80 & 4 & - & 4 & 100 \\
\hline Hillsboro gas well & 25 Nov. 2011 & 5 & 5 & 100 & 5 & - & 5 & 100 \\
\hline Hill C cemet. Sp. & 30 Mar. 2012 & 5 & 5 & 100 & 5 & - & 5 & 100 \\
\hline unknown & Unknown & 5 & 5 & 100 & 5 & - & 5 & 100 \\
\hline Grayson Co RR & 28 Feb. 2012 & 5 & 5 & 100 & 5 & - & 5 & 100 \\
\hline unknown & 21 Mar. 2012 & 5 & 4 & 80 & 4 & - & 4 & 100 \\
\hline Dorchester RR B4B8 & 15 Nov. 2011 & 5 & 5 & 100 & 5 & - & 5 & 100 \\
\hline Dorchester field2 & 15 Nov. 2011 & 5 & 4 & 80 & 3 & 1 & 4 & 100 \\
\hline Hillsboro old airport & 25 Nov. 2011 & 5 & 4 & 80 & 4 & - & 4 & 100 \\
\hline unknown & Unknown & 5 & 5 & 100 & 5 & - & 5 & 100 \\
\hline Vermont & & 60 & 46 & 77 & 38 & 0 & 38 & 83 \\
\hline Addison (B) & 18 Sept. 2012 & 7 & 6 & 86 & 6 & - & 6 & 100 \\
\hline Orleans $(\mathrm{J})$ & 7 Sept. 2012 & 11 & 9 & 82 & 9 & - & 9 & 100 \\
\hline Orleans (J) & 21 Sept. 2012 & 12 & 6 & 50 & 3 & 0 & 3 & 50 \\
\hline Orleans (J) & 4 Oct. 2012 & 9 & 7 & 78 & 7 & - & 7 & 100 \\
\hline Grand Isle (R) & 27 Sept. 2012 & 15 & 14 & 93 & 9 & 0 & 9 & 64 \\
\hline Grand Isle (R) & 9 Oct. 2012 & 6 & 4 & 67 & 4 & - & 4 & 100 \\
\hline
\end{tabular}

DNA samples were also tested from 100 visually identified as non-Hessian flies from different traps, and all these non-Hessian fly samples were negative with both CM and HFSM markers.

varied among the locations: $98 \%$ for the Kansas samples, $60 \%$ for the Oklahoma samples, $93 \%$ for the Texas samples, and $77 \%$ for the Vermont samples. The lower success rates for the Oklahoma and Vermont samples might be due to the fact that insects were in poorer conditions on these pheromone traps, which might have affected DNA extraction efficiency.

Insect DNA samples positive to the common marker were then analyzed with the Hessian fly-specific markers HFSM1 and HFSM2 (Table 2). Nearly 100\% 
of the insect samples that were positive for CM were identified as Hessian fly using the specific markers for the samples from Kansas, Oklahoma, and Texas. A lower percent (83\%) of CM-positive samples from Vermont were identified by the two Hessian fly-specific markers to be Hessian fly males. The lower percent for the samples from Vermont might be due to poorer sample preservation that made it difficult to exclude non-Hessian fly insects visually before the samples were subjected to marker analysis. Alternatively, Hessian flies from Vermont contain sequence variations in the primer-targeted regions of the two target genes, resulting in failure of PCR amplification. Further research is needed to distinguish these possibilities. When a combination of HFSM1 and HFSM2 was used after a positive CM result, a 97\% positive result was obtained. HFSM1 alone also provided a $95 \%$ positive result with the CM-positive samples. HFSM2 was not tested extensively with pheromone-trapped insects, but high percentages of positive detection were obtained with a limited number of trap insects and with known laboratory Hessian flies. Thus, even though we designed two Hessian fly-specific markers, one marker may be actually enough for identifying Hessian flies from other insects in pheromone traps.

In summary, we have established a simple molecular marker-based method for more accurate identification of Hessian fly males captured in sticky pheromone traps than visual examination. The method should also be useful for identification of larvae, pupae, and female adults of the Hessian fly when the identity of these stages is in doubt, such as pupae found in wheat grains or insects in hay in America for export. As Hessian fly populations evolve rapidly, the usefulness of the established protocol in other countries remains to be verified. Because DNA testing for Hessian fly identification requires specialized skills and equipment, it is not realistic to expect that this method would be suitable for common use by farmers or pest managers. However, our results have confirmed that around $90 \%$ of insects on sticky pheromone traps that are identified as Hessian fly males using readily seen morphological characteristics are indeed Hessian fly males. Our data support that the use of Hessian fly sticky pheromone traps is a reliable approach for documenting activity in wheat fields in wheat fields. Our results underscore the importance of conducting immediate visual examination of insects on sticky pheromone traps soon after they are collected from the field. This can be seen from the differences between Kansas and Vermont fly-identification results (Table 2 ). Insects on sticky pheromone traps from Kansas were visually examined immediately after the traps were collected, whereas insects on traps from Vermont were stored at $-20^{\circ} \mathrm{C}$ for several weeks before visual examination. More than $96 \%$ of insects identified based on morphology as Hessian fly males from the Kansas sample were verified as Hessian fly males according to the marker analysis. In comparison, only $83 \%$ of the insects visually identified as Hessian fly males from the Vermont sample were confirmed by marker analysis as Hessian fly males. Appendages and wings were found to be dislocated for some insects on the Vermont traps, making it difficult to make correct visual identification. In addition, storage of the insects on traps in freezers also made it more difficult to obtain whole insects from the trap for DNA extraction. The quality and quantity of DNA samples might have also played a role in lower PCR identification rates in the Vermont samples.

\section{Acknowledgments}

We thank Carlos Campos, TX A\&M Agrilife Extension, for field and laboratory analysis with Hessian fly sticky pheromone traps. This research was partially supported by the grant USDA-NIFA-RAMP \#2010-51101-21642. Mention of trade names or commercial products in this publication is solely for the purpose of providing specific information and does not imply recommendation or endorsement by the U.S. Department of Agriculture. USDA is an equal-opportunity provider and employer.

\section{References Cited}

Anderson, K. M., Y. Hillbur, J. Reber, B. Hanson, R. O. Ashley, and M. O. Harris. 2012. Using sex pheromone trapping to explore threats to wheat from Hessian fly (Diptera: Cecidomyiidae) in the Upper Great Plains. J. Econ. Entomol. 105: 1988-1997.

Andersson, M. N., J. Haftmann, J. J. Start, S. E. Cambron, M. O. Harris, S. P. Foster, S. Franke, W. Francke, and Y. Hillbur. 2009. Identification of sex pheromone components of the Hessian fly, Mayetiola destructor. J. Chem. Ecol. 35: 81-95.

Bouktila, D., M. Mezghani, M. Marrakchi, H. Makni. 2006. Genetic variation and relatedness in Tunisian wheat midges of the genus Mayetiola (Diptera: Cecidomyiidae), inferred from biological and molecular data. Acta Entomol. Sin. 49: 822-828.

Buntin, G. D. 1992. Assessment of a microtube injection system for applying systemic insecticides at planting for Hessian fly control in winter wheat. Crop Prot. 11: 366370.

Buntin, G. D. 1999. Hessian fly (Diptera: Cecidomyiidae) injury and loss of winter wheat grain yield and quality. J. Econ. Entomol. 92: 1190-1197.

Buntin, G. D., and P. L. Bruckner. 1990. Effect of planting date on Hessian fly infestation and production of triticale. Appl. Agric. Res. 5: 82-88.

Buntin, G. D., P. L. Bruckner, J. W. Johnson, J. E. Foster. 1990. Effectiveness of selected genes for Hessian fly resistance in wheat. J. Agric. Entomol. 7: 283-291.

Buntin, G. D., S. L. Ott, and J. W. Johnson. 1992. Integration of plant resistance, insecticides, and planting date for management of the Hessian fly (Diptera: Cecidomyiidae) in winter wheat. J. Econ. Entomol. 85: 530-538.

Byers, R. A., and R. L. Gallun. 1971. Ability of the Hessian fly to stunt winter wheat. I. Effect of larval feeding on elongation of leaves. J. Econ. Entomol. 65: 955-958.

Chen, M. S., E. Echegaray, J. Whitworth, H. Wang, P. Sloderbeck, A. Knutson, K. Giles, and T. Royer. 2009. Virulence analysis of Hessian fly populations from Texas, Oklahoma, and Kansas. J. Econ. Entomol. 102: 774-780.

Chen, M. S., X. M. Liu, Z. Yang, H. Zhao, R. H. Shukle, J. J. Stuart, and S. Hulbert. 2010. Unconventional conservation among genes encoding small secreted salivary gland proteins from a gall midge. BMC Evol. Biol. 10: 296. 
Chen, H., Y. C. Zhu, R. J. Whitworth, J. C. Reese, and M. S. Chen. 2013. Serine and cysteine protease-like genes in the genome of a gall midge and their interactions with host plant genotypes. Insect Biochem. Mol. Biol. 43: 701-711.

Gagne, R. J. 1975. A new species of Mayetiola (Diptera Cecidomyiidae) on American beachgrass in New Jersey. Entomol. News 86: 85-87.

Gould, F. 1998. Sustainability of transgenic insecticidal cultivars: Integrating pest genetics and ecology. Annu. Rev. Entomol. 43: 701-726.

Johnson, A. J., P. G. Weintraub, R. Katoch, B. J. Schemerhorn, and R. H. Shukle. 2012. Biological and molecular characterization of Hessian fly (Diptera: Cecidomyiidae) from Israel. Bull. Entomol. Res. 102: 632-643.

Liu, X. L., J. Bai, L. Huang, L. Zhu, X. Liu, N. Weng, J. C. Reese, M. Harris, J. J. Stuart, and M. S. Chen. 2007. Gene expression of different wheat genotypes during attack by virulent and avirulent Hessian fly (Mayetiola destructor) larvae. J. Chem. Ecol. 33: 2171-2194.
Morgan, G., C. Sansone, and A. Knutson. 2005. The Hessian Fly in Texas wheat. E-350. Texas AgriLife Extension Bull. (http:/ / agrilifebookstore.org).

PaDIL-Plant Biosecurity Toolbox. 2010. Diagnostic Methods for Hessian Fly Mayetiola destructor. (http://old. padil.gov.au/pbt/index.php?q=node/164).

Pauly, P. J. 2002. Fighting the Hessian fly. Environ. Hist. 7: 385-507.

Sosa, O. J. 1978. Biotype L, ninth biotype of the Hessian fly. J. Econ. Entomol. 71: 458-460.

Stuart, J. J., M. S. Chen, M. O. Harris, and R. H. Shukle. 2012. Gall Midges (Hessian Flies) as Plant Pathogens. Annu. Rev. Phytopathol. 50: 339-357.

Whitworth, R. J., P. E. Sloderbeck, and H. N. Davis. 2010. Crop insects in Kansas. Kans. State Univ. Agric. Exp. Stn. Coop. Ext. Serv. 152: 133.

Received 4 September 2013; accepted 12 March 2014. 